\title{
An Early Study on the Mechanisms that Allow Tissue-Engineered Vascular Grafts to Resist Intimal Hyperplasia
}

\author{
Heather L. Prichard • Roberto J. Manson • \\ Louis DiBernardo • Laura E. Niklason • \\ Jeffrey H. Lawson • Shannon L. M. Dahl
}

Received: 31 March 2011 / Accepted: 26 June 2011 /Published online: 12 July 2011

(C) The Author(s) 2011. This article is published with open access at Springerlink.com

\begin{abstract}
Intimal hyperplasia is one of the prominent failure mechanisms for arteriovenous fistulas and arteriovenous access grafts. Human tissue-engineered vascular grafts (TEVGs) were implanted as arteriovenous grafts in a novel baboon model. Ultrasound was used to monitor flow rates and vascular diameters throughout the study. Intimal hyperplasia in the outflow vein of TEVGs was assessed at the anastomosis and at juxta-anastomotic regions via histological analysis, and was compared to intimal hyperplasia with polytetrafluoroethylene (PTFE) grafts in the baboon model and in literature reports from other animal models. Less venous intimal hyperplasia was observed in histological sections with arteriovenous TEVGs than with arteriovenous PTFE grafts. TEVGs were associated with a mild, noninflammatory intimal hyperplasia. The extent of intimal tissue that formed with TEVG placement correlated with the rate of blood flow through tissue engineered
\end{abstract}

H. L. Prichard · S. L. M. Dahl $(\bowtie)$

Humacyte, Inc.,

PO Box 12695, Durham, NC 27709, USA

e-mail: dahl@humacyte.com

R. J. Manson

Department of Surgery, Duke University Medical Center,

Durham, NC, USA

L. DiBernardo

Department of Pathology, Duke University Medical Center,

Durham, NC, USA

L. E. Niklason

Departments of Anesthesiology and Biomedical Engineering,

Yale University,

New Haven, CT, USA

J. H. Lawson

Departments of Surgery and Pathology,

Duke University Medical Center,

Durham, NC, USA vascular grafts at 2 weeks postimplant. Outflow vein dilatation was observed with increased flow rate. Both mid-graft flow rates and outflow vein diameters reached a plateau by week 4 , which suggested that venous remodeling and intimal hyperplasia largely occurred within the first 4 weeks of implant in the baboon model. Given their compliant and noninflammatory nature, TEVGs appear resistant to triggers for venous intimal hyperplasia that are common for PTFE arteriovenous grafts, including (1) abundant proinflammatory macrophage populations that are associated with PTFE grafts and (2) compliance mismatch between PTFE grafts and the outflow vein. Our findings suggest that arteriovenous TEVGs develop only a mild form of venous intimal hyperplasia, which results from the typical hemodynamic changes that are associated with arteriovenous settings.

Keywords Tissue-engineered vascular graft · Intimal hyperplasia $\cdot$ Arteriovenous graft $\cdot$ Remodeling $\cdot$ Blood flow rate $\cdot$ Noninflammatory

\section{Introduction}

There are more than 347,000 patients on chronic hemodialysis [1]. Each year, more than 100,000 new end stage renal disease patients begin therapy on hemodialysis [1]. Native arteriovenous fistulas have recently been the first choice for access, but even with the best surgical care, fistula prevalence is only $47 \%$ in the USA [2]. The remainder of access is dependent on arteriovenous grafts or catheters, with a preference for grafts when possible [3]. Currently, most arteriovenous grafts that are placed for hemodialysis access are comprised of synthetic polytetrafluoroethylene (PTFE). Synthetic arteriovenous grafts suffer from significant drawbacks, which include a high rate of 
infection (9\% per annum) and a propensity for occlusion due to thrombosis and intimal hyperplasia $(40-60 \%$ in the first year), with a median patency of only 10 months [4-7]. PTFE grafts require frequent interventions to maintain patency throughout their lifetime, which necessitates costly surgical intervention, increased overall healthcare costs, and increased patient morbidity $[8,9]$.

By some reports, up to $85 \%$ of PTFE graft failures result from intimal hyperplasia at either the venous anastomosis or outflow vein [10]. Intimal hyperplasia is typically characterized by intimal thickening of the vein, which diminishes the vein lumen, decreases blood flow rate, may lead to postcannulation bleeding due to intragraft pressurization, and eventually leads to thrombosis and occlusion [11]. The intima is defined as the innermost layer of an artery or vein, on the luminal side of the internal elastic lamina. In a healthy vein, the intima is one cell layer thick and comprised solely of endothelial cells. With intimal hyperplasia, cells may migrate into the intima from the medial and adventitial layers of the vein and from the circulation $[10,12,13]$. Once they migrate into the intima, these cells express markers for myofibroblast and smooth muscle cell phenotypes. The cells proliferate and deposit extracellular matrix proteins $[10,14]$. The formation of new tissue in the intima can compromise blood flow.

Intimal hyperplasia is thought to be triggered by the high wall shear stresses created by AV grafts [15], and compliance mismatch between PTFE and the native vein [11]. Intimal hyperplasia also has been identified in regions with turbulent flow, flow separation, low shear stress, and oscillatory shear [15]. In addition, PTFE's synthetic composition commonly leads to inflammation at the graft placement site following implant, which promotes intimal hyperplasia formation [10].

Development of tissue-engineered vascular grafts may provide a new opportunity for hemodialysis patients. If designed correctly, a tissue-engineered graft has the potential to reduce incidence of failure mechanisms associated with PTFE grafts. We have recently described a new human tissue-engineered vascular graft (TEVG) with compliance between that of human vein and human artery [16], which obviates concern about compliance mismatch. Furthermore, our TEVGs are made of extracellular matrix proteins, which make them highly biocompatible and resistant to inflammation [16]. These TEVGs are acellular, which allows them to be stored in simple refrigeration for up to 1 year, thereby making them readily available to patients at the time of need [16]. We demonstrated excellent function of these TEVGs in multiple vascular settings. TEVGs placed as arteriovenous grafts in a baboon model for 1-6 months demonstrated a patency rate of $88 \%$, without incidence of graft infection [16].

In this study, we evaluate intimal hyperplasia formation associated with arteriovenous placement of TEVGs. We also investigate the impact of flow rate and outflow vein remodeling on intimal hyperplasia formation.

\section{Materials and Methods}

\section{Formation of TEVGs}

Human TEVGs were made as described previously [16]. Briefly, human aortic smooth muscle cells were isolated from donor aortas that were consented for research use. Cells were seeded onto tubular polyglycolic acid felt scaffolds $(6 \mathrm{~mm}$ inner diameter) and strained cyclically $(2.5 \%$ at $2.75 \mathrm{~Hz})$ [17] in a bioreactor to produce grafts. The medium for growth of human TEVGs was high glucose Dulbecco's modified Eagle's medium with $20 \%$ serum, $0.13 \mathrm{U} / \mathrm{ml}$ insulin, $10 \mathrm{ng} / \mathrm{ml}$ basic fibroblast growth factor, $0.5 \mathrm{ng} / \mathrm{ml}$ epidermal growth factor, $10,000 \mathrm{U} / \mathrm{ml}$ penicillin $\mathrm{G}, 3 \mathrm{ng} / \mathrm{ml}$ copper sulfate, $50 \mathrm{ng} / \mathrm{ml}$ L-proline, $40 \mathrm{ng} / \mathrm{ml} \mathrm{L}$-alanine, and $50 \mathrm{ng} / \mathrm{ml}$ glycine, and was changed thrice weekly. L-Ascorbic acid was added thrice weekly to TEVG cultures.

After 10 weeks of culture, TEVGs were decellularized as described previously [18] in phosphate-buffered saline with $0.12 \mathrm{M}$ sodium hydroxide, $1 \mathrm{M}$ sodium chloride, and $25 \mathrm{mM}$ EDTA, containing either $8 \mathrm{mM}$ CHAPS or $1.8 \mathrm{mM}$ sodium dodecyl sulfate. TEVGs were exposed to each detergent solution for up to $6 \mathrm{~h}$ at room temperature and were then washed with phosphate-buffered saline. All TEVGs were decellularized prior implantation. Decellularized TEVGs were stored at $4^{\circ} \mathrm{C}$ in phosphate buffered saline without calcium or magnesium.

\section{Animal Use}

All procedures were approved by Duke University's Animal Care and Use Committee. Baboons received humane care according to the "Guide for the Care and Use of Laboratory Animals" [19]. All surgical procedures were performed in sterile fashion under general anesthesia. After each surgery, graft patency was confirmed, wounds were closed, and animals were recovered. Baboons were anticoagulated with heparin at implant $(100 \mathrm{U} / \mathrm{kg}$ given intravenously followed by $1,000 \mathrm{U} / \mathrm{h}$ for the duration of the procedure) and received aspirin $(10 \mathrm{mg} / \mathrm{kg}$ ) daily preoperatively until the end of the study.

An old world primate model was chosen to provide phylogenetic similarity to humans, which allowed implantation of noncross-linked human matrix-containing grafts without immunosuppression. Adult male baboons (Papio Anubis, $20-30 \mathrm{~kg}$ ) are physically large enough to support implantation of a $6 \mathrm{~mm}$ inner diameter TEVG in a clinically relevant anatomic setting. 
Surgical Implantation Techniques

The surgical portion of this study has been described elsewhere for TEVGs [16]. Briefly, nine adult male baboons underwent arteriovenous placement of human TEVGs $(6 \mathrm{~mm}$ inner diameter). One TEVG was placed between the aorta and the vena cava for 1 month. Eight TEVGs were placed for up to 6 months between the axillary artery and distal brachial vein in the upper arms of baboons, which provided a superficial site amenable for simulating hemodialysis access at 1,3 , and 6 months [16]. Arteriovenous PTFE grafts were also placed in the baboon model between the aorta and vena cava (one graft for 1 month) and between the axillary artery and distal brachial vein in the upper arm (one graft for 6 months). All animals received only one graft to minimize risk of heart failure. All anastomoses were created with a running 6-0 prolene suture technique. One TEVG remained in vivo at the time of analysis. Two other animals with upper arm TEVGs were excluded from histological evaluation in this study. In one animal, technical difficulties with access resulted in prolonged manual pressure on the TEVG, which led to graft occlusion, unrelated to intimal hyperplasia. The other animal was excluded after pulling open its surgical incision site, exposing the TEVG and creating a wound infection.

\section{Duplex Ultrasound}

In the baboon upper arm model, duplex ultrasound was used to monitor diameter $(D)$ and maximum velocity $\left(V_{\max }\right)$ preoperatively for native vessels, postoperatively for the graft, and then again at 2, 4, 12, and 24 weeks for patent grafts. Flow rates in the aorta-cava model were not measured since grafts were not accessible to ultrasound. Flow rate $(Q)$ was calculated as

$Q=\left(V_{\max } / 2\right)(\pi)(D / 2)^{2}$

\section{Histology}

Tissues were fixed in 10\% neutral buffered formalin, embedded in paraffin, sliced (5- $\mu \mathrm{m}$ sections), and stained with hematoxylin and eosin (H\&E) or Movat's. Immunostaining was performed for $\alpha$-smooth muscle actin (SMC and myofibroblast marker; Dako M0851, 1:400 dilution), CD31 (endothelial cell marker; Dako M0823, 1:700 dilution), and CD68 (macrophage marker; Thermo MS397-P1, 1:400 dilution), using 3,3'-diaminobenzidine stain. All were counterstained with hematoxylin.

Morphometric Analysis of Intimal Hyperplasia

Venous anastomotic sections and juxta-anastomotic sections of the outflow vein were analyzed for formation of intimal hyperplasia. Figure 1 demonstrates sectioning of upper arm grafts. Aorta-cava grafts were sectioned in similar fashion. Images were taken using an Olympus BX41 microscope with a mounted Olympus DP25 camera. CellSens standard imaging software was used for morphometric analysis. Sections stained with Movat's were used for analysis because the presence of elastin in the medial layer of the vein allowed for clear identification of the intima. The internal elastic lamina was used to define the transition between the media and the intima. First, the area of intimal hyperplasia (AI) was measured in veins at the anastomotic sites and in juxta-anastomotic outflow vein sections. Second, the underlying length of vein was measured, which, before intimal thickening, was the lumen length (LL). Intimal thicknesses (IT) were calculated as the total area of intimal hyperplasia divided by the length of the underlying tissue (IT=AI/LL) [20]. The thickness of the medial layer was measured in similar fashion, using the medial area (MA) and the underlying length, which was the length of the interface (LI) between the medial layer and the adventitial layer. Medial thickness (MT) was then calculated as MT $=$ MA/LI. The ratio of intimal thickness to medial thickness was then IT/MT [21]. The ratio of intimal area to medial area was AI/MA.

\section{Statistical Analysis}

Statistical analyses were performed with a Student's twosample $t$ test, assuming unequal variances. Correlations were assessed using the Pearson method. Two-sided $P<$ 0.05 indicated statistical significance. Numeric values are presented as the mean \pm standard error of the mean.

\section{Results}

Placement of TEVGs as Arteriovenous Grafts in a Baboon Model Triggers Mild, Nonaggressive Intimal Hyperplasia

Histology demonstrated that arteriovenous TEVGs in a baboon model had substantially less intimal hyperplasia at

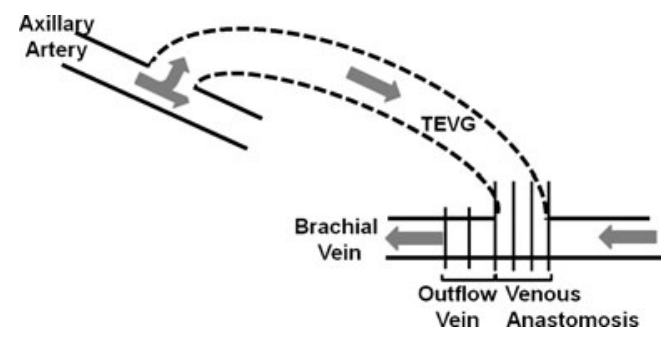

Fig. 1 Schematic of TEVG placement, and locations of histological sections taken for assessment of intimal hyperplasia in the brachial vein 
the venous anastomosis than arteriovenous PTFE grafts. Minimal intimal hyperplasia was observed at the venous anastomosis with all TEVGs in the baboon model (representative image shown in Fig. 2a). The extent of intimal hyperplasia shown in Fig. 2a for TEVGs is substantially less than that typically shown in the literature for clinical PTFE grafts (Fig. 2b). Representative high magnification images show venous intimal hyperplasia after 6 months in a baboon model for both TEVGs (Fig. 2c) and PTFE grafts (Fig. 2d). Notably less intimal hyperplasia was observed with TEVGs.

Quantification of intimal hyperplasia further demonstrated that TEVGs were associated with less aggressive intimal hyperplasia than PTFE grafts in the baboon model (Table 1). Intimal and medial areas of the vein were quantified (1) at the venous anastomosis and (2) in the outflow vein proximal to the anastomosis, for both TEVGs ( $6 \mathrm{~mm}$ diameter) and PTFE grafts (6 mm diameter) in the baboon model. A ratio of intimal to medial area was presented to allow benchmarking to literature reports of PTFE grafts ( $5 \mathrm{~mm}$ diameter) in a porcine arteriovenous (carotid artery to jugular vein) model [11] (Table 1). In the baboon model, we observed that intimal hyperplasia formed most readily at the venous anastomosis and dissipated rapidly along the outflow vein for PTFE grafts. Thus, in the baboon model, we noted substantial differences in intimal hyperplasia between TEVGs and PTFE grafts at the venous anastomosis, but not in the outflow vein. In the porcine model, quantification of intimal hyperplasia with PTFE grafts clearly demonstrates aggressive intimal hyperplasia at only 8 weeks at both the
Table 1 Ratio of intimal area to medial area

\begin{tabular}{lll}
\hline $\begin{array}{l}\text { Arteriovenous } \\
\text { access creation } \\
\text { method }\end{array}$ & $\begin{array}{l}\text { Ratio of intimal area to } \\
\text { medial area at venous } \\
\text { anastomosis }\end{array}$ & $\begin{array}{l}\text { Ratio of intimal } \\
\text { area to medial area } \\
\text { in outflow vein }\end{array}$ \\
\hline $\begin{array}{c}\text { TEVGs in a baboon } \\
\text { model }\end{array}$ & $0.37 \pm 0.10$ & $0.18 \pm 0.10$ \\
$\begin{array}{c}\text { PTFE grafts in a } \\
\text { baboon model }\end{array}$ & $1.3 \pm 0.6$ & $0.11 \pm 0.01$ \\
$\begin{array}{c}\text { PTFE grafts in a } \\
\text { porcine model }\end{array}$ & 0.87 & 0.33 \\
\hline
\end{tabular}

${ }^{\mathrm{a}}$ From Rotmans et al. [11]

venous anastomosis and the juxta-anastomotic outflow vein [11] (Table 1). Thus, quantitative analysis and histological observations suggest that TEVGs may prompt less intimal hyperplasia than PTFE grafts, especially at anastomotic sections at the graft/vein interface.

Cell populations in venous intimal hyperplasia in anastomotic sections were determined via immunostaining (Fig. 3). Actin-positive cells were identified throughout the intimal tissue (Fig. 3a). Actin-positive cells are typically observed in intimal hyperplasia associated with arteriovenous fistulae or arteriovenous PTFE grafts [14].

CD-31-positive cells (endothelial cells) were observed on the luminal surface, but were not observed within the intimal tissue (Fig. 3b). Others have shown microvessel formation within venous intima that is defined by positive staining for endothelial cell markers [10]. Furthermore, microvessel formation indicates an aggressive type of intimal hyperplasia [22]. Thus, the absence of microvessel
Fig. 2 Venous intimal hyperplasia is minimal at 3 months for TEVGs in a baboon model. a Venous anastomosis of a TEVG explanted at 3 months ( $G$ and $V$ denote the graft and vein, respectively). b Venous anastomosis of a PTFE graft explanted from a human (PTFE image courtesy of Dr. Prabir RoyChaudhury, modified and reprinted with permission from Kelly et al. [22]). High magnification of the shoulder region of the venous anastomosis of $\mathbf{c}$ a TEVG at 6 months in the baboon model and $\mathbf{d}$ a PTFE graft at 6 months in the baboon model. Arrows in c and d indicate the interface between the media and intima, with the intima positioned below the media
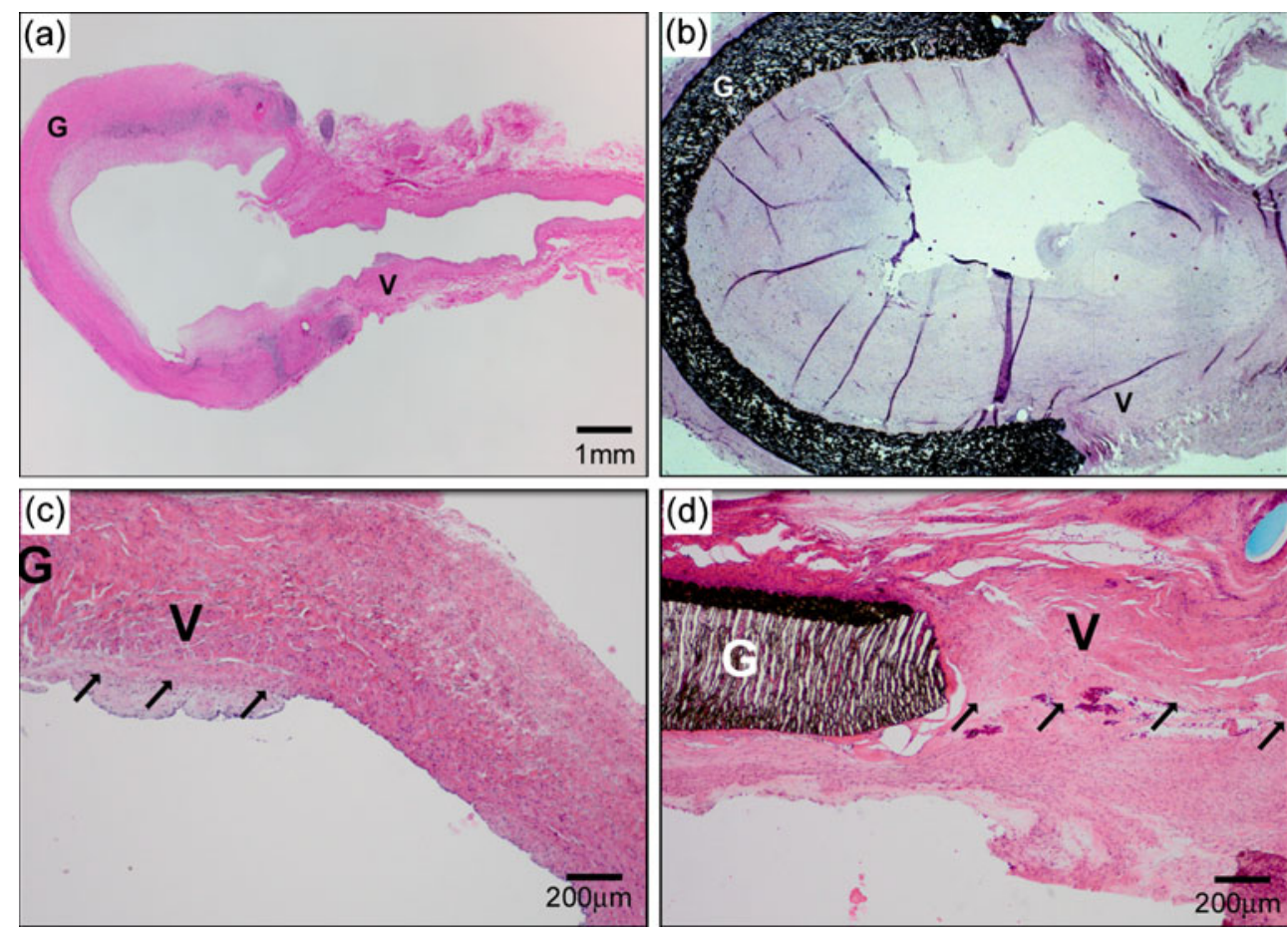


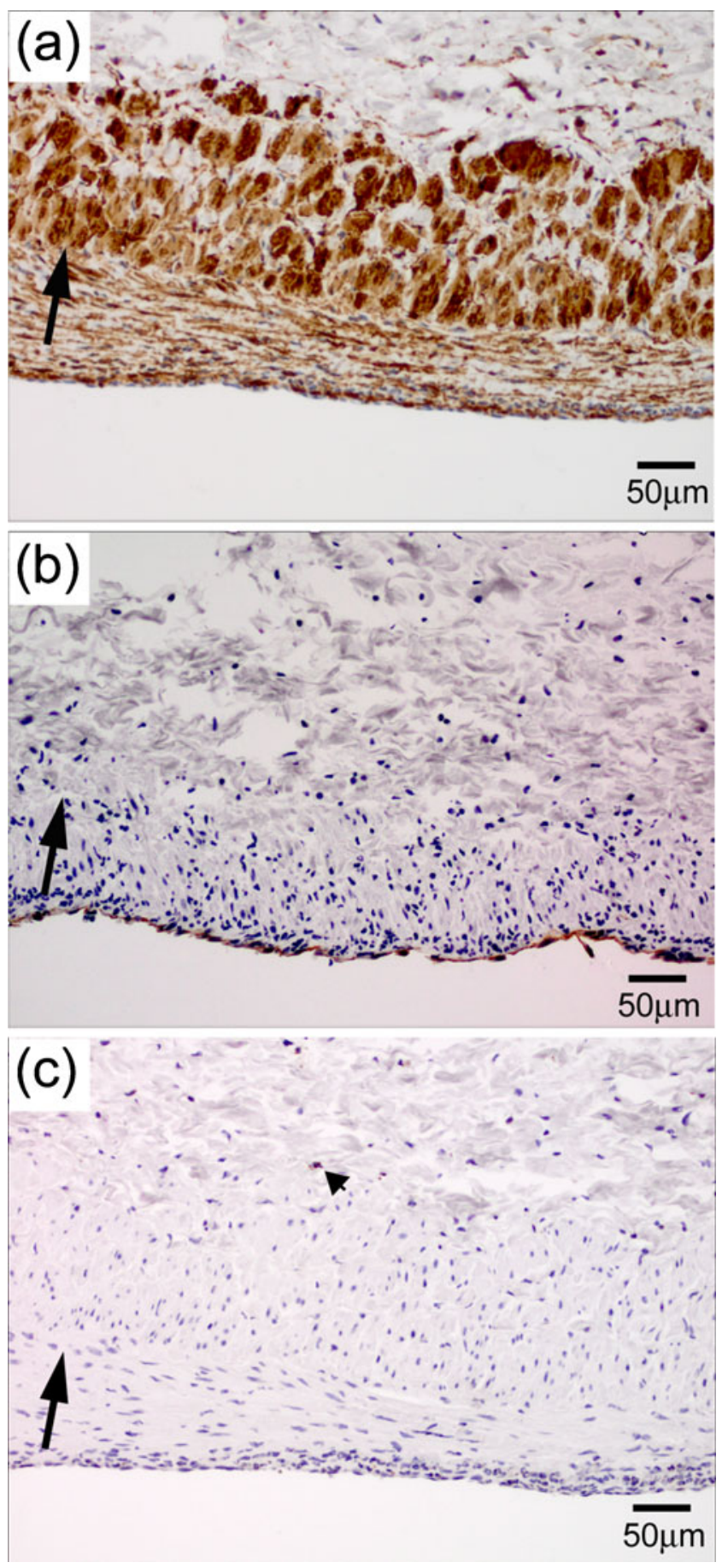

Fig. 3 Immunostaining of venous intimal hyperplasia that is associated with arteriovenous TEVGs. Large arrows indicate the interface between the media and intima, with the intima positioned below the media in all panels. a Alpha-smooth muscle actin positive cells (stain brown) are observed in the media and in the intima. b CD31-positive cells (positive cells stain brown; blue nuclear counterstain) were observed on the luminal surface but not within the intima. c CD68-positive cells (positive cells stain brown; blue nuclear counterstain) were sparse (small arrow points to the only cell that stains positively for CD68 in this field of view, located in the media of the vein). Representative 6-month images are shown formation within intimal tissue in this study suggests that TEVGs prompt a nonaggressive form of intimal hyperplasia.

CD-68-positive cells (macrophages) were very sparse (Fig. 3c). In contrast, a substantial macrophage population has been observed within intimal tissue adjacent to PTFE arteriovenous grafts [10]. The prominent macrophage population that infiltrates PTFE grafts releases cytokines and growth factors and thereby promotes intimal hyperplasia $[14,23,24]$. Thus, the inflammatory characteristic of synthetic PTFE is thought to increase the aggressiveness of intimal hyperplasia. TEVGs likely have substantially less macrophage-related stimulation of intimal hyperplasia than PTFE.

The extent of intimal hyperplasia formation was not significantly different between 3 and 6 month explants (Table 2, $P=0.3$ ). Although this nonaggressive intimal hyperplasia did not appear to worsen with time, we observed variation in the extent of intimal hyperplasia between grafts (note high values for standard error of the mean). In contrast, other groups have demonstrated that PTFE grafts trigger intimal hyperplasia that grows substantially with time $[11,25]$. The observation that intimal hyperplasia did not worsen significantly between 3 and 6 months led us to study variability between TEVG recipients, as variability is always observed in the clinic and in animal models.

The Mild Intimal Hyperplasia that Forms with Arteriovenous TEVG Placement is Influenced by Host Blood Flow Rate and Venous Adaptation to Flow Rate

The goal of placing arteriovenous grafts for hemodialysis access is to increase blood flow above $300 \mathrm{ml} / \mathrm{min}$ to allow for successful hemodialysis [26]. Implantation of arteriovenous grafts allows blood to flow from the artery to the vein with diminished resistance. The new path of decreased resistance, from the artery to vein via the graft, minimizes the impact of back pressure from tapering distal arteries on arterial flow rate and thereby allows flow to increase.

As anticipated, placement of TEVGs as arteriovenous grafts in the baboon upper arm model increased flow rates (Fig. 4a). Preoperative flow rates for the inflow artery and outflow vein were $301 \pm 85$ and $68 \pm 13 \mathrm{ml} / \mathrm{min}$, respectively.

Table 2 Intimal hyperplasia at 3 and 6 month explants

\begin{tabular}{lcc}
\hline & $\begin{array}{l}\text { Ratio of intimal area to } \\
\text { medial area at venous } \\
\text { anastomosis }\end{array}$ & $\begin{array}{l}\text { Ratio of intimal area } \\
\text { to medial area in } \\
\text { outflow vein }\end{array}$ \\
\hline $\begin{array}{l}\text { TEVGs explanted at } \\
3 \text { months }\end{array}$ & $0.57 \pm 0.35$ & $0.38 \pm 0.38$ \\
$\begin{array}{l}\text { TEVGs explanted at } \\
6 \text { months }\end{array}$ & $0.30 \pm 0.03$ & $0.11 \pm 0.05$ \\
\hline
\end{tabular}



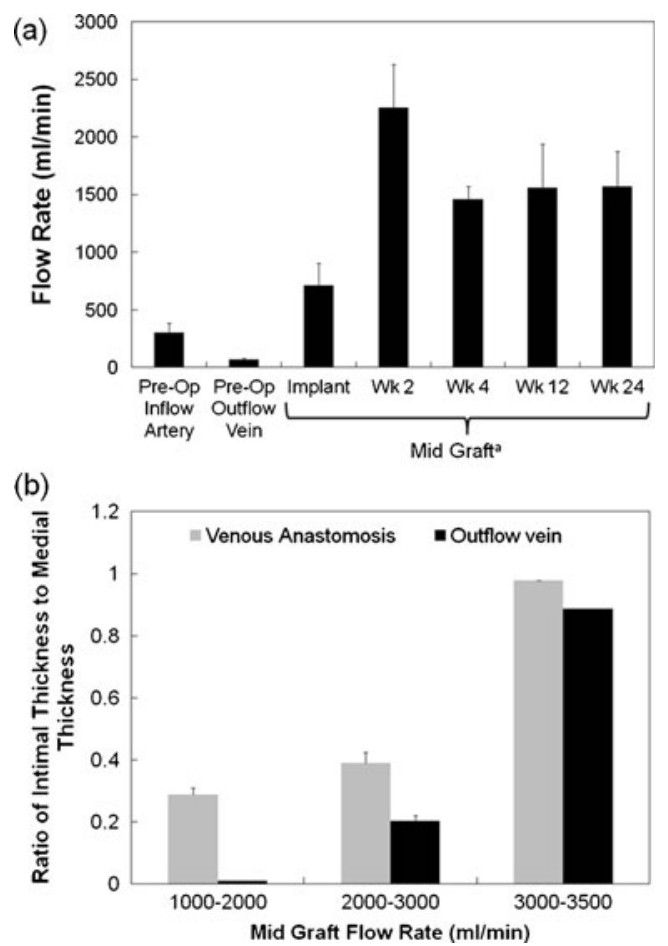

Fig. 4 Flow rates. a Flow before and after placement of TEVGs as arteriovenous grafts (Pre-op preoperative, Wk week). Superscript letter a modified and reprinted with permission from Dahl et al. [16]. b Flow rate at week 2 impacted the extent of intimal growth

Mid-graft flow reached $709 \pm 195 \mathrm{ml} / \mathrm{min}$ immediately after implant. Flow rates through TEVGs increased further by week $2(P=0.009$, Fig. $4 \mathrm{a})$, with a range of $1,081-3,127 \mathrm{ml} / \mathrm{min}$ at 2 weeks. Mid-graft flow through the PTFE graft in the baboon upper arm model was $963 \mathrm{ml} / \mathrm{min}$ at 2 weeks. Mid-graft flow through TEVGs displayed a decreasing trend between weeks 2 and $4(P=$ $0.06)$ and reached a steady-state level of flow thereafter (Fig. 4a; no significant differences were detected between flow rates at 4,12 , and 24 weeks, $P>0.2$ ).

Intimal hyperplasia has been shown to be triggered by high wall shear stresses that accompany high flow rates [15]. Others have observed that flow rates decrease as intimal hyperplasia progresses [11]. Therefore, we considered the hypothesis that (1) the high week 2 flow rates trigger intimal hyperplasia and (2) the absence of a decrease in flow rate after week 4 may indicate that intimal thickening occurred primarily within the first 4 weeks of implant.

At week 2, TEVG mid-graft flow rates positively correlated with the extent of intimal hyperplasia formation in the vein (Fig. 4b). Specifically, higher flow rates at week 2 were associated with an increase in the ratio of intimal thickness to medial thickness in the vein at explant, both in anastomotic and in juxta-anastomotic sections $(P=0.04$ for correlation between flow rate at week 2 and venous anastomosis thickness ratio, and $P=0.02$ for correlation between flow rate and juxta-anastomotic outflow vein thickness ratio). Our findings for TEVGs are in agreement with a clinical study, which demonstrated that week 2 velocity was correlated with the extent of venous intimal hyperplasia formation triggered by arteriovenous PTFE grafts [27].

No significant correlations were observed between intimal thickening and flow rate through TEVGs at any time points other than week $2(P>0.3$ for weeks $0,4,12$, and 24). The lack of a correlation between flow rate and intimal hyperplasia beyond week 4 suggests that much of the intimal thickening may have occurred largely within the first 4 weeks of implant. Time-course assessments of intimal hyperplasia with PTFE grafts in a porcine model have shown substantial intimal growth in histological sections by week 2 and another increase in intimal growth between weeks 2 and 4 [11], which supports the ability of intimal hyperplasia to form in this early time frame.

Evaluation of outflow vein diameter further supports our hypothesis that intimal thickening occurs largely within the first month of implant and then reaches a plateau. We observed outflow vein dilatation within 2 weeks of TEVG placement (Fig. 5, $P=0.0007$ ). Outflow vein diameter decreased between weeks 2 and $4(P=0.03)$ and reached a steady-state level thereafter (no significant differences between weeks 4,12 , and $24, P>0.4$ ).

Given that outflow vein dilatation is thought to be prompted by the increase in shear stress that accompanies an increase in flow rate [28] and that veins dilate in an attempt to return shear stress levels back to normal [29], it is logical that our diameter observations match the temporal trend presented for flow rate in Fig. 4a. This agrees with one of the few clinical studies that has tracked flow and dilatation using ultrasound, wherein increases in flow rate led to venous dilatation in fistulas [30].

The observed decrease in diameter between weeks 2 and 4 (Fig. 5) likely correlates with a period of active intimal thickening. Given that diameter did not decrease after week

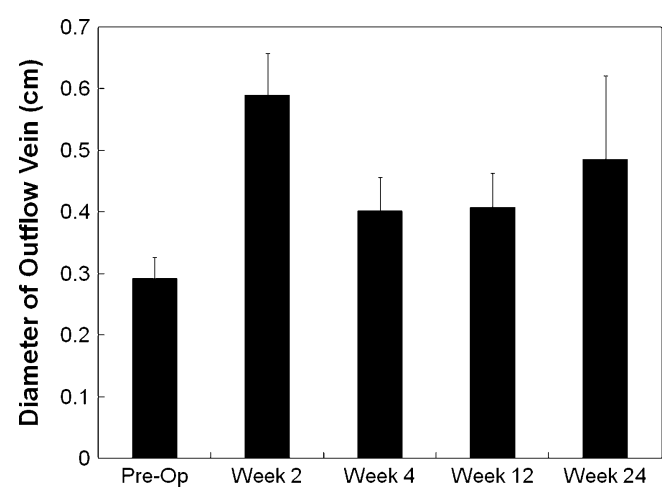

Fig. 5 Outflow vein diameters changed after placement of TEVGs as arteriovenous grafts 
4, we submit that venous intimal thickening occurred mostly within 4 weeks of TEVG implant. Note that there was no statistical correlation between preoperative vein diameter and intimal hyperplasia formation, which suggests that flow-ratemediated remodeling, and not preoperative anatomy, dictated intimal hyperplasia formation.

\section{Discussion}

TEVGs have compliances within the range of human artery and vein and have been shown to be noninflammatory in vivo [16]. In this study, we demonstrate that the venous intimal hyperplasia that forms in response to arteriovenous placement of TEVGs in a baboon model is only a mild type of intimal hyperplasia, as indicated by less intimal hyperplasia formation than observed with PTFE grafts, the absence of intimal microvessel formation, and a very sparse macrophage population. Furthermore, this mild intimal hyperplasia did not progress between 3 and 6 months. For TEVGs, we conclude that the extent of venous intimal tissue formation was likely driven by early changes in flow rate. High flow rates may be associated with high shear stress, turbulent flow, flow separation, and oscillatory shear. Additionally, we suggest that the intimal hyperplasia associated with TEVGs largely forms within the first month of implant and does not grow aggressively thereafter.

In contrast, PTFE grafts have been shown to be proinflammatory and thereby become infiltrated with macrophages $[14,23]$. The intimal hyperplasia that forms with placement of PTFE arteriovenous grafts is aggressive, as demonstrated by microvessel formation and continued growth with time [10, $11,22]$. This ongoing growth is likely related to PTFE's proinflammatory nature, with a large macrophage population that continuously triggers intimal hyperplasia [14, 23, 24]. It is important to note that early PTFE flow rates in the baboon upper arm model and mean PTFE flow rates in the porcine neck model $(1,065 \pm 469 \mathrm{ml} / \mathrm{min}$ at 4 weeks [11]) were close to the lowest flow rates observed in TEVGs. Thus, the observation that PTFE grafts triggered more aggressive intimal hyperplasia than TEVGs was likely due to PTFE's inflammatory nature. The compliance of PTFE $(1.6 \%$ per $100 \mathrm{mmHg}$ [31]) is only half that of TEVGs (3.3\% per $100 \mathrm{mmHg}$ [16]), and therefore, compliance mismatch may also be a contributor toward increased intimal hyperplasia with PTFE grafts. Mechanisms for venous intimal hyperplasia formation with TEVGs and PTFE grafts are proposed in Fig. 6.

In addition to posing a problem for arteriovenous grafts, intimal and medial hyperplasia is also a significant problem in arteriovenous fistulas [21]. Some triggers for formation of intimal hyperplasia in arteriovenous fistulas differ from those in PTFE grafts. Unlike PTFE grafts, fistulas do not have a large macrophage population to stimulate intimal formation. However, venous intimal thickening with fistu-

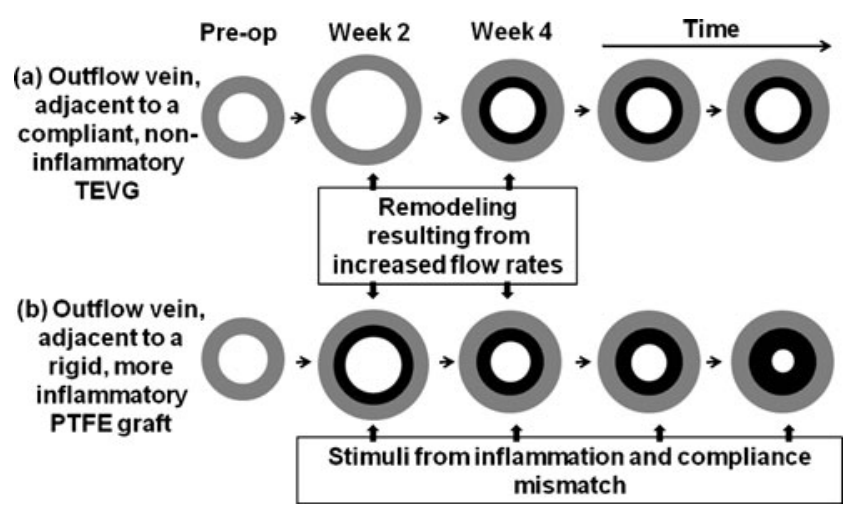

Fig. 6 Proposed mechanisms for outflow vein intimal hyperplasia formation with arteriovenous placement of TEVGs and PTFE grafts

las has been shown to be stimulated in part by expression of growth factors within the artery [32].

TEVGs are acellular at implant, and the absence of cells may aid in resistance to intimal hyperplasia. While we have not performed any analysis for presence or absence of growth factors in human TEVGs, it is possible that acellular TEVGs present less growth factor expression than a native artery, thereby making the outflow vein less susceptible to intimal hyperplasia. Furthermore, the absence of cells at early time points makes acellular TEVGs reasonably inert during the early implant phase, where dynamic changes in flow are prevalent. ATEVG containing cells might respond to high shear stresses via cellular proliferation, forming intimal tissue within the first month of implant when flow rates and shear stresses are at their highest. The absence of cells in TEVGs at the time of implant may thereby aid its ability to resist early cell-mediated intimal injury.

The impact of host blood flow rate on intimal hyperplasia in the vein suggests that future TEVGs (and PTFE grafts) might better control venous intimal hyperplasia if the grafts have a mechanism to limit flow rate at the graft exits. Such a design might prevent exposure of excessive flow rates to the venous anastomosis and may thereby reduce the vein's intimal thickening response. Tapered PTFE grafts have been evaluated to restrict flow, with the goal of reducing steal, but have not demonstrated definitive functional improvements in the clinic [33]. Thus, an alternate approach to coarctation of the graft likely will be necessary to confirm the hypothesis that high flow rates lead to more intimal hyperplasia and address the flowrelated mechanism of intimal hyperplasia formation.

Several animal studies in other labs have focused on controlling flow rate or shear stress to determine their impact on intimal hyperplasia formation, yet these studies have been performed primarily in the arterial circulation or the venous circulation, but not in arteriovenous models. In the arterial circulation, increasing blood flow across preformed intimal tissue led to regression of the intima in a baboon model [34]. In 
the venous setting, extremely low shear stress has been shown to promote intimal hyperplasia in a rabbit model [35]. Substantial differences in hemodynamics exist between bypass and arteriovenous grafts [15], and therefore, it is difficult to extrapolate these findings to the arteriovenous setting.

The study described herein has several known limitations. First, this is an early study with a small number of subjects. The trends observed are sensible, but we plan to continuously monitor these trends in future animals as more data on arteriovenous TEVG implantation is acquired. Second, animals were treated with aspirin throughout the course of this study, which has been shown to decrease intimal hyperplasia [36]. However, aspirin therapy is recommended clinically [37] and is used routinely in preclinical models that study intimal hyperplasia, including those referenced as benchmarks in this study [11, 22, 25].

The baboon model had clear benefits for evaluation of a human vascular graft. Baboons are old world primates, which are phylogenetically closer to humans than more common cardiovascular large animal models, such as pigs, dogs, and sheep. The concordant baboon model abrogated risk of xenogenic immune response to a human cardiovascular implant. Furthermore, the vascular anatomy of baboons was large enough to support implantation of a $6 \mathrm{~mm}$ diameter graft. In addition, the clotting and fibrinolytic systems of primates are more closely related to humans [38]. Therefore, baboons served as the best model for human-based cardiovascular implants. In contrast, PTFE grafts are frequently evaluated in animal models that are affordable and widely available (e.g., pigs) because PTFE is not at risk of triggering a xenogenic immune response.

The baboon model, however, also had clear limitations. Primates are significantly more expensive to purchase and maintain than any other animal [39], are difficult to handle and maintain [40], and are limited in availability. In order to circumvent the issues of high costs and limited animal availability, we considered implanting a PTFE graft in the contralateral arm of each animal. After careful consideration, we did not pursue this strategy because implantation of two high-flow arteriovenous grafts in each animal would lead to a substantial increase in cardiac output, which would create a real risk of heart failure. These limitations constrained our ability to evaluate a large number of PTFE control grafts, so only two control grafts were implanted. Therefore, in addition to presenting data on these two control PTFE grafts in the baboon model, we have presented data from literature reports of arteriovenous PTFE grafts in porcine animal models.

\section{Conclusions}

The results of this study provide a useful framework with which we can think about intimal hyperplasia formation.
The correlation between early flow rates and intimal formation may also translate to venous intima development with fistulas and PTFE grafts. Only a handful of studies have histologically analyzed intimal hyperplasia in fistulas and PTFE grafts, yet these studies are primarily focused on failed arteriovenous grafts or fistulas [10, 21, 23] since functioning access sites are not explanted. Furthermore, failed access sites are rarely removed. Thus, the insights provided by this study can contribute the body of knowledge regarding progression of intimal hyperplasia and its triggers.

Use of TEVGs in a baboon arteriovenous model led to mild, nonaggressive intimal hyperplasia that likely formed within 1 month of implant. TEVGs are noninflammatory and compliant, and thereby avoid two common triggers of arteriovenous PTFE grafts for progression of intimal hyperplasia. Histological assessments demonstrated that TEVGs had less intimal hyperplasia than PTFE grafts. TEVGs that resist substantial intimal hyperplasia could possibly provide a new treatment option for patients with end stage renal disease. Both reduced incidence of intimal hyperplasia failure and slowed progression of intimal hyperplasia-related failure could lead to a decrease in surgical intervention of arteriovenous access grafts. Decreased surgical intervention, in turn, could decrease overall end stage renal disease healthcare costs and patient morbidity.

Acknowledgments We would like to acknowledge Taylor Hensley and Letia Dean for assistance with histological staining, and Terry Williams for performing ultrasound measurements. This study was funded by Humacyte, Inc.

Conflict of interest H.L.P. and S.L.M.D. are employed by Humacyte. R.J.M., J.H.L., L.D., and L.E.N. have either been paid as consultants to Humacyte or have a financial interest in Humacyte.

Open Access This article is distributed under the terms of the Creative Commons Attribution Noncommercial License which permits any noncommercial use, distribution, and reproduction in any medium, provided the original author(s) and source are credited.

\section{References}

1. US Renal Data System. (2010). USRDS 2010 annual data report: Atlas of chronic kidney disease and end-stage renal disease in the United States. Bethesda: National Institutes of Health, National Institute of Diabetes and Digestive and Kidney Diseases.

2. DOPPS. (2009). 2009 annual report of the dialysis outcomes and practice patterns study: Hemodialysis data 1999-2008. Ann Arbor: Arbor Research Collaborative for Health.

3. National Kidney Foundation. (2006). NKF KDOQI guidelines. Retrieved from http://www.kidney.org/PROFESSIONALS/kdoqi/ guideline_upHD_PD_VA/va_guide1.htm.

4. Schild, A., Perex, E., Gillaspie, E., Seaver, C., Livingstone, J., \& Thibonnier, A. (2008). Arteriovenous fistulae vs. arteriovenous 
grafts: a retrospective review of 1,700 consecutive vascular access cases. The Journal of Vascular Access, 9, 231-235.

5. Cinat, M., Hopkins, J., \& Wilson, S. (1999). A prospective evaluation of PTFE graft patency and surveillance techniques in hemodialysis access. Annals of Vascular Surgery, 13, 191-198.

6. Helling, T., Nelson, P., \& Shelton, L. (1992). A prospective evaluation of plasma-TFE and expanded PTFE grafts for routine and early use as vascular access during hemodialysis. Annals of Surgery, 216, 596-599.

7. Zibari, G., Gadallah, M., Landreneau, M., McMillan, R., Bridges, R., Costley, K., et al. (1997). Preoperative vancomycin prophylaxis decreases incidence of postoperative hemodialysis vascular access infections. American Journal of Kidney Diseases, 30, 343-348.

8. Roy-Chaudhury, P., Sukhatme, V. P., \& Cheung, A. K. (2006). Hemodialysis vascular access dysfunction: a cellular and molecular viewpoint. Journal of the American Society of Nephrology, 17(4), $1112-1127$.

9. Lok, C. (2007). Fistula first initiative: advantages and pitfalls. Clinical Journal of the American Society of Nephrology, 2(5), 1043-1053.

10. Roy-Chaudhury, P., Kelly, B. S., Miller, M. A., Reaves, A., Armstrong, J., Nanayakkara, N., et al. (2001). Venous neointimal hyperplasia in polytetrafluoroethylene dialysis grafts. Kidney International, 59(6), 2325-2334.

11. Rotmans, J. I., Velema, E., Verhagen, H. J., Blankensteijn, J. D., Kastelein, J. J., de Kleijn, D. P., et al. (2003). Rapid, arteriovenous graft failure due to intimal hyperplasia: a porcine, bilateral, carotid arteriovenous graft model. Journal of Surgical Research, 113(1), 161-171.

12. Caplice, N. M., Wang, S., Tracz, M., Croatt, A. J., Grande, J. P., Katusic, Z. S., et al. (2007). Neoangiogenesis and the presence of progenitor cells in the venous limb of an arteriovenous fistula in the rat. American Journal of Physiology. Renal Physiology, 293 (2), F470-F475.

13. Shi, Y., O'Brien, J. E., Fard, A., Mannion, J. D., Wang, D., \& Zalewski, A. (1996). Adventitial myofibroblasts contribute to neointimal formation in injured porcine coronary arteries. Circulation, 94(7), 1655-1664.

14. Roy-Chaudhury, P., Wang, Y., Krishnamoorthy, M., Zhang, J., Banerjee, R., Munda, R., et al. (2009). Cellular phenotypes in human stenotic lesions from haemodialysis vascular access. Nephrology, Dialysis, Transplantation, 24(9), 2786-2791.

15. Haruguchi, H., \& Teraoka, S. (2003). Intimal hyperplasia and hemodynamic factors in arterial bypass and arteriovenous grafts: a review. Journal of Artificial Organs, 6(4), 227-235.

16. Dahl, S. L., Kypson, A. P., Lawson, J. H., Blum, J. L., Strader, J. T., Li, Y., et al. (2011). Readily available tissue-engineered vascular grafts. Science Translational Medicine, 3(68), 68ra69.

17. Niklason, L. E., Gao, J., Abbott, W. M., Hirschi, K., Houser, S., Marini, R., et al. (1999). Functional arteries grown in vitro. Science, 284, 489-493.

18. Dahl, S. L. M., Koh, J., Prabhakar, V., \& Niklason, L. E. (2003). Decellularized native and engineered arterial scaffolds for transplantation. Cell Transplantation, 12, 659-666.

19. National Research Council. (2009). Guide for the care and use of laboratory animals (8th ed.). Washington: The National Academies Press.

20. Lumsden, A. B., Chen, C., Coyle, K. A., Ofenloch, J. C., Wang, J. H., Yasuda, H. K., et al. (1996). Nonporous silicone polymer coating of expanded polytetrafluoroethylene grafts reduces graft neointimal hyperplasia in dog and baboon models. Journal of Vascular Surgery, 24(5), 825-833.

21. Roy-Chaudhury, P., Arend, L., Zhang, J., Krishnamoorthy, M., Wang, Y., Banerjee, R., et al. (2007). Neointimal hyperplasia in early arteriovenous fistula failure. American Journal of Kidney Diseases, 50(5), 782-790.

22. Kelly, B. S., Heffelfinger, S. C., Whiting, J. F., Miller, M. A., Reaves, A., Armstrong, J., et al. (2002). Aggressive venous neointimal hyperplasia in a pig model of arteriovenous graft stenosis. Kidney International, 62(6), 2272-2280.

23. Li, L., Terry, C., Shiu, Y., \& Cheung, A. (2008). Neointimal hyperplasia associated with synthetic hemodialysis grafts. Kidney International, 74(10), 1247-1261.

24. Mattana, J., Effiong, C., Kapasi, A., \& Singhal, P. (1997). Leukocyte-polytetrafluoroethylene interaction enhances proliferation of vascular smooth muscle cells via tumor necrosis factoralpha secretion. Kidney International, 52(6), 1478-1485.

25. Baig, K. (2003). A porcine model of intimal-medial hyperplasia in polytetrafluoroethylene arteriovenous grafts. The Journal of Vascular Access, 4, 111-117.

26. Dixon, B. (2006). Why don't fistulas mature? Kidney International, 70(8), 1413-1422.

27. Hofstra, L., Bergmans, D. C., Leunissen, K. M., Hoeks, A. P., Kitslaar, P. J., Daemen, M. J., et al. (1995). Anastomotic intimal hyperplasia in prosthetic arteriovenous fistulas for hemodialysis is associated with initial high flow velocity and not with mismatch in elastic properties. Journal of the American Society of Nephrology, 6(6), 1625-1633.

28. Roy-Chaudhury, P., Spergel, L. M., Besarab, A., Asif, A., \& Ravani, P. (2007). Biology of arteriovenous fistula failure. Journal of Nephrology, 20(2), 150-163.

29. Malovrh, M. (2010). Non-matured arteriovenous fistulae for haemodialysis: diagnosis, endovascular and surgical treatment. Bosnian Journal of Basic Medical Sciences, 10(Suppl 1), S13-S17.

30. Corpataux, J. M., Haesler, E., Silacci, P., Ris, H. B., \& Hayoz, D. (2002). Low-pressure environment and remodelling of the forearm vein in Brescia-Cimino haemodialysis access. Nephrology, Dialysis, Transplantation, 17(6), 1057-1062.

31. Kidson, I. G. (1983). The effect of wall mechanical properties on patency of arterial grafts. Annals of the Royal College of Surgeons of England, 65(1), 24-29.

32. Rodriguez, V. M., Grove, J., Yelich, S., Pearson, D., Stein, M., \& Pevec, W. C. (2002). Effects of brachytherapy on intimal hyperplasia in arteriovenous fistulas in a porcine model. Journal of Vascular and Interventional Radiology, 13(12), 1239-1246.

33. Dammers, R., Planken, R. N., Pouls, K. P., Van Det, R. J., Burger, H., Van Der Sande, F. M., et al. (2003). Evaluation of 4-mm to 7-mm versus 6-mm prosthetic brachial-antecubital forearm loop access for hemodialysis: results of a randomized multicenter clinical trial. Journal of Vascular Surgery, 37(1), 143-148.

34. Mattsson, E. J., Kohler, T. R., Vergel, S. M., \& Clowes, A. W. (1997). Increased blood flow induces regression of intimal hyperplasia. Arteriosclerosis, Thrombosis, and Vascular Biology, 17(10), 2245-2249.

35. Meyerson, S. L., Skelly, C. L., Curi, M. A., Shakur, U. M., Vosicky, J. E., Glagov, S., et al. (2001). The effects of extremely low shear stress on cellular proliferation and neointimal thickening in the failing bypass graft. Journal of Vascular Surgery, 34(1), 90-97.

36. McCann, R. L., Hagen, P. O., \& Fuchs, J. C. (1980). Aspirin and dipyridamole decrease intimal hyperplasia in experimental vein grafts. Annals of Surgery, 191(2), 238-243.

37. Osborn, G., Escofet, X., \& Da Silva, A. (2008). Medical adjuvant treatment to increase patency of arteriovenous fistulae and grafts. Cochrane Database of Systematic Reviews, (4):86.

38. Mason, R. G., \& Read, M. S. (1971). Some species differences in fibrinolysis and blood coagulation. Journal of Biomedical Materials Research, 5, 121-128.

39. Rashid, S. T., Salacinski, H. J., Hamilton, G., \& Seifalian, A. M. (2004). The use of animal models in developing the discipline of cardiovascular tissue engineering: a review. Biomaterials, 25(9), $1627-1637$.

40. Narayanaswamy, M., Wright, K. C., \& Kandarpa, K. (2000). Animal models for atherosclerosis, restenosis, and endovascular graft research. Journal of Vascular and Interventional Radiology, 11, 5-17. 